\title{
On the convergence behavior of recursive adaptive noise cancellation structure in the presence of crosstalk
}

\author{
Pascal Scalart ${ }^{\# 1}$ and Ludovick Lepauloux ${ }^{* 2}$ \\ \# ENSSAT / University of Rennes - INRIA \\ 6 rue de Kérampont, 22305 Lannion Cedex, France \\ ${ }^{1}$ pascal.scalart@univ-rennesl.fr \\ * Orange Labs - TECH/OPERA \\ 2 Avenue Pierre Marzin, 22307 Lannion Cedex, France \\ ${ }^{2}$ ludovick. lepauloux@me.com
}

\begin{abstract}
In this paper, we address the problem of determining the equilibrium point of a feedback structure for two-channel adaptive noise cancellation in the presence of crosstalk. We focus on an important characteristic of adaptive filters, namely the steady-state mean-square error that remains after the algorithm has converged but independently of the considered algorithm. Our approach relies on analysis of the relationships between the desired signals and their artifacts (distortion, residual noise) at the system outputs. We show that the equilibrium state is obtained when the energy of the distortion on the output signals is the same on each channel. Using this equilibrium state, we provide answers to questions for which no satisfactory answers are currently available for this structure. Examples are given to illustrate that even qualitatively, these answers can be good approximations. Results of simulations sustain our claims.
\end{abstract}

\section{INTRODUCTION}

Analysis of convergence and stability for adaptive algorithms can pose difficult problems but are crucial in adaptive signal processing. As for adaptive noise cancelling or blind source separation, convergence properties and asymptotic behavior of the feedback structure for two-channel adaptive noise cancellation in the presence of crosstalk have been studied in very simple cases, see [1] for example. Most of the time, stationary input signals or single tap mixing filters were assumed. This is mainly due to the heavy complexity of the considered separation structure (feedback structure), illustrated by Fig. 1. Indeed, the cross-coupling in the separation structure introduces an IIR filter which leads to potential instability. Thus, the convergence analysis is essential but more difficult than in adaptive FIR filtering. More precisely and in comparison with the Hessian matrix in adaptive FIR filtering, the Hessian matrix associated with the considered structure is time-varying even when the input signals are stationary. However, in [2] and [3] a complete analysis of the recursive structure is provided for self-adaptive separation of convolutively mixed signals. Based on Robbins-Monro type algorithm, this study was carried out only around the desired solution, which is assumed to be the equilibrium point corresponding to the separating solutions.

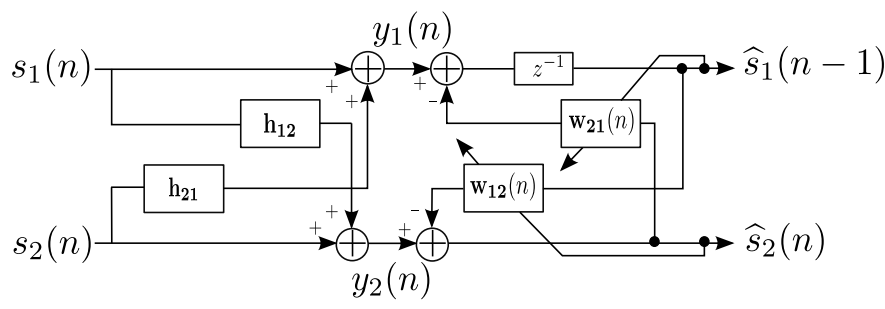

Fig. 1. Mixing model (left) and separation structure (right). The introduced delay arbitrarily fixed in the separation structure is justified by implementation considerations. It can be put indifferently on $\widehat{s}_{1}$ or $\widehat{s}_{2}$, it depends on which filter is first adapted.

On account of ordinary differential equation technique, equilibrium and stability conditions can be analyzed for a large class of separating functions that are restricted in order to ensure the separating point is an equilibrium state. All of these results were obtained for algorithms with asymptotically constant step-size allowing handling of non-stationary systems, especially those that are slowly varying in time, which we interested in. Though the ordinary differential equation approach to convergence analysis is a powerful technique, it does not prove convergence of the algorithm to the global minimum, except for convex error surface. Unfortunately, as shown in [4], when considering a minimum energy criterion for each output channel of Fig. 1, i.e. by minimizing $E\left[\widehat{s}_{1}^{2}\right]$ and $E\left[\widehat{s}_{2}^{2}\right]$, the two error surfaces associated with each adaptive filters $\mathbf{w}_{12}$ and $\mathbf{w}_{\mathbf{2 1}}$ are linear, bilinear, and quadratic forms of vectors $\mathbf{w}_{12}$ and $\mathbf{w}_{\mathbf{2 1}}$, respectively, with coefficients of the quadratic forms that are linear and quadratic in vectors $\mathbf{w}_{12}$ and $\mathbf{w}_{21}$ [4], [5], [6]. Consequently, multiple local minima may appear. Thus, similarly to technique for adaptive algorithms for blind source separation, additional assumptions are necessary in order to guarantee that there are no local minima and consequently, that asymptotic convergence to the desired solution is ensured.

This paper is organized as follows. In section II, we first 
introduce the separation model. Then in section III, in contrast with the usual approaches consisting of first defining the adaptive algorithm and then analyzing its behavior according to a given structure, in this article, we first define, analytically, the equilibrium point of the separation structure of Fig. 1 independently of the considered algorithms. Our derivation relies on energy conservation arguments and it does not restrict the data to being Gaussian or white. Next, we derive several useful properties of the considered structure. Extensive simulations confirm the derived results and prove the validity of this equilibrium state.

\section{PRoblem Statement}

\section{A. Advantage of the recursive structure}

Let us consider the mixing model described in Fig. 1. The two observations can be expressed in the Z-domain as:

$$
\left[\begin{array}{l}
Y_{1}(z) \\
Y_{2}(z)
\end{array}\right]=\boldsymbol{M}(z)\left[\begin{array}{l}
S_{1}(z) \\
S_{2}(z)
\end{array}\right], \quad \boldsymbol{M}(z)=\left[\begin{array}{cc}
1 & H_{21}(z) \\
H_{12}(z) & 1
\end{array}\right]
$$

Where $\boldsymbol{M}(z)$ denotes the mixing matrix. In practice, the mixing filters are unknown and have to be estimated. In order to cancel the existing coupling effect between the observations and to recover the original sources, the recursive structure can be used. The main advantage of this structure in contrast with the feedforward one is that it does not need to estimate the inverse of the mixing matrix $\boldsymbol{M}(z)$, noted $\boldsymbol{G}(z)$ and defined as

$$
\boldsymbol{G}(z)=\frac{1}{1-H_{12}(z) H_{21}(z)}\left[\begin{array}{cc}
1 & -H_{21}(z) \\
-H_{12}(z) & 1
\end{array}\right] .
$$

Indeed, the separating filters $\mathbf{w}_{\mathbf{i j}}$ just have to 'mimic' the filter $\mathbf{h}_{\mathrm{ij}}$ of the mixing model, i.e. to converge towards them. Then, inversion is actually performed by the recurrent connection in the structure, so that the overall separating system eventually implements $\boldsymbol{G}(z)$.

\section{B. Limitations of the recursive structure}

A closed look on the input-ouput relationship (1) and (2) of the recursive structure, where $L$ is the filter length, shows that it is impossible to implement this method without delay. Indeed, each output $\widehat{s}_{i}$ at time index $n$ depends on the other one at the same time index.

$$
\begin{aligned}
& \widehat{s}_{1}(n)=y_{1}(n)-\sum_{i=0}^{L} w_{21}(i) y_{2}(n-i) \\
& \widehat{s}_{2}(n)=y_{2}(n)-\sum_{j=0}^{L} w_{12}(j) y_{1}(n-1-j)
\end{aligned}
$$

Furthermore, the recursive structure imposes natively two constraints that impact on the nature of the adaptive and mixing filters. First, due to the dependence between the outputs, the mixing and adaptive filters must be, at least, causal. In [7], Al-Kindi and Dunlop choose to place a unit delay in each adaptive filter to compensate for the delay in the feedback paths. Secondly, practical considerations impose that, at least, one of the mixing filters be strictly causal, since the introduced delay doesn't permit to identify the zero order coefficient of the associated mixing filter. Consequently, the corresponding observation-output relationship in the $\mathrm{Z}$-domain is given by

$$
\begin{aligned}
{\left[\begin{array}{c}
\widehat{S}_{1}(z) \\
\widehat{S}_{2}(z)
\end{array}\right]=\frac{1}{1-W_{12}(z) W_{21}(z) z^{-1}} \times } & {\left[\begin{array}{cc}
1 & -W_{21}(z) \\
-W_{12}(z) z^{-1} & 1
\end{array}\right]\left[\begin{array}{l}
Y_{1}(z) \\
Y_{2}(z)
\end{array}\right] . }
\end{aligned}
$$

From a theoretical point of view, this requirement enables the filters $\mathbf{w}_{\mathbf{i j}}$ to reach the optimal solutions. In addition, this causality constraint is not restrictive, especially when considering room acoustic impulse responses which are strictly causal.

\section{EQUILIBRIUM STATE}

In the following, uppercase symbols in boldface font denote matrix quantity whereas lowercase symbols in boldface font indicate a vector quantity. Normal font is used for scalar quantity. The diag operator denotes diagonal elements of matrix, $*$ the convolution operator and ${ }^{T}$ the transpose operator. As stated in Figure 1, $\left\{s_{1}, s_{2}\right\}$ and $\left\{y_{1}, y_{2}\right\}$ are respectively the source and the observation signals. We use $\left\{\widehat{s}_{1}, \widehat{s}_{2}\right\}$ to represent the estimated signals.

\section{A. Distortion analysis on each output}

According to the mixing and separation models, the output signal distortion $\Delta s_{1}(n)=\widehat{s_{1}}(n)-s_{1}(n)$ on $s_{1}(n)$ can be formulated as

$$
\begin{aligned}
\Delta & s_{1}(n)=y_{1}(n)-\mathbf{w}_{\mathbf{2 1}}{ }^{T}(n) \widehat{\mathbf{s}}_{\mathbf{2}}(n)-s_{1}(n) \\
=\mathbf{h}_{\mathbf{2 1}}{ }^{T} \mathbf{S}_{\mathbf{2}}(n)- & \mathbf{W}_{\mathbf{2 1}}{ }^{T}(n) \mathbf{y}_{\mathbf{2}}(n)+ \\
& \mathbf{W}_{\mathbf{2 1}}{ }^{T}(n) \operatorname{diag}\left\{\mathbf{W}_{\mathbf{1 2}}{ }^{T}(n) \widehat{\mathbf{M}}_{\mathbf{1}}(n-1)\right\}
\end{aligned}
$$

with

$$
\begin{aligned}
& \mathbf{h}_{\mathbf{2 1}}=\left[h_{21}^{0} h_{21}^{1} \ldots h_{21}^{L-1}\right]^{T}, \\
& \mathbf{w}_{\mathbf{2 1}}(n)=\left[w_{21}^{0}(n) w_{21}^{1}(n) \ldots w_{21}^{L-1}(n)\right]^{T}, \\
& \mathbf{y}_{\mathbf{2}}(n)=\left[y_{2}(n) y_{2}(n-1) \ldots y_{2}(n-L+1)\right]^{T} \text {, } \\
& \mathbf{s}_{\mathbf{2}}(n)=\left[s_{2}(n) s_{2}(n-1) \ldots s_{2}(n-L+1)\right]^{T}, \\
& \widehat{\mathbf{s}_{2}}(n)=\left[\widehat{s_{2}}(n) \widehat{s_{2}}(n-1) \ldots \widehat{s_{2}}(n-L+1)\right]^{T} \text {, } \\
& \widehat{\mathbf{M}}_{\mathbf{1}}(n-1)=\left[\widehat{\mathbf{s}}_{\mathbf{1}}(n-1) \widehat{\mathbf{s}}_{\mathbf{1}}(n-2) \ldots \widehat{\mathbf{s}}_{\mathbf{1}}(n-L)\right], \\
& \mathbf{W}_{12}(n)=\left[\mathbf{w}_{12}(n) \mathbf{w}_{12}(n-1) \ldots \mathbf{w}_{12}(n-L+1)\right] .
\end{aligned}
$$

Assuming (A1) that the filter coefficients are slowly varying in time [6], i.e. $\mathbf{w}_{\mathbf{1 2}}(n-j)=\mathbf{w}_{\mathbf{1 2}}(n) \quad \forall j=0, \ldots, L-1$, translates into fixing a small step-size (asymptotic behaviour) and noting that $\mathbf{y}_{\mathbf{2}}(n)=\mathbf{s}_{\mathbf{2}}(n)+\mathbf{M}_{\mathbf{1}}^{T}(n) \mathbf{h}_{\mathbf{1 2}}$, the relation (3) can be reformulated as

$$
\begin{aligned}
& \Delta s_{1}(n)=\Delta \mathbf{h}_{\mathbf{2 1}}{ }^{T}(n) \mathbf{s}_{\mathbf{2}}(n)- \\
& \quad \mathbf{w}_{\mathbf{2 1}}^{T}(n)\left[\mathbf{M}_{\mathbf{1}}^{T}(n) \mathbf{h}_{\mathbf{1 2}}-\widehat{\mathbf{M}}_{\mathbf{1}}^{T}(n-1) \mathbf{w}_{\mathbf{1 2}}(n)\right] .
\end{aligned}
$$


Where $\Delta \mathbf{h}_{\mathbf{2 1}}(n)=\mathbf{h}_{\mathbf{2 1}}-\mathbf{w}_{\mathbf{2 1}}(n)$ is the misalignment vector and $\mathbf{M}_{\mathbf{1}}(n)=\left[\mathbf{s}_{\mathbf{1}}(n) \mathbf{s}_{\mathbf{1}}(n-1) \ldots \mathbf{s}_{\mathbf{1}}(n-L+1)\right]$ the matrix of the source signals. Introducing the matrix $\Delta \mathbf{S}_{\mathbf{1}}(n)=\mathbf{M}_{\mathbf{1}}(n)-$ $\widehat{\mathbf{M}}_{\mathbf{1}}(n)$, the expression in the bracket can be expressed such that

$$
\begin{aligned}
& \mathbf{M}_{\mathbf{1}}^{T}(n) \mathbf{h}_{\mathbf{1 2}}-\widehat{\mathbf{M}}_{\mathbf{1}}^{T}(n-1) \mathbf{w}_{\mathbf{1 2}}(n)= \\
& \quad \mathbf{M}_{\mathbf{1}}^{T}(n) \mathbf{h}_{\mathbf{1 2}}-\left[\mathbf{M}_{\mathbf{1}}^{T}(n-1)-\Delta \mathbf{S}_{\mathbf{1}}^{T}(n-1)\right] \mathbf{w}_{\mathbf{1 2}}(n)
\end{aligned}
$$

with

$$
\begin{aligned}
& \mathbf{M}_{\mathbf{1}}^{T}(n) \mathbf{h}_{\mathbf{1 2}}-\mathbf{M}_{\mathbf{1}}^{T}(n-1) \mathbf{w}_{\mathbf{1 2}}(n)= \\
& \quad \sum_{i=0}^{L-1} \mathbf{s}_{\mathbf{1}}(n-i) h_{12}(i)-\sum_{j=0}^{L-1} \mathbf{s}_{\mathbf{1}}(n-1-j) w_{12}(j) .
\end{aligned}
$$

Imposing (A2) causality constraint on $\mathbf{h}_{\mathbf{1 2}}$ such that $h_{12}(0)=$ 0 and $w_{12}(L-1)=0$, the expression (5) leads to

$$
\begin{aligned}
& \mathbf{M}_{\mathbf{1}}{ }^{T}(n) \mathbf{h}_{\mathbf{1 2}}-\mathbf{M}_{\mathbf{1}}^{T}(n-1) \mathbf{w}_{\mathbf{1 2}}(n)= \\
& \quad \sum_{i=1}^{L-1} \mathbf{s}_{\mathbf{1}}(n-i) h_{12}(i)-\sum_{j=0}^{L-2} \mathbf{s}_{\mathbf{1}}(n-1-j) w_{12}(j) \\
& \quad=\sum_{k=0}^{L-2} \mathbf{s}_{\mathbf{1}}(n-1-k)\left[h_{12}(k+1)-w_{12}(k)\right] \\
& =\widetilde{\mathbf{M}}_{\mathbf{1}}(n-1)\left(\widetilde{\mathbf{h}}_{\mathbf{1 2}}-\widetilde{\mathbf{w}}_{\mathbf{1 2}}(n)\right),
\end{aligned}
$$

where $\widetilde{\mathbf{M}}_{\mathbf{1}}(n-1)$ is a truncated version of $\mathbf{M}_{\mathbf{1}}(n-1)$ of size $L \times(L-1)$. Similarly, $\widetilde{\mathbf{h}}_{\mathbf{1 2}}=\left.\mathbf{h}_{\mathbf{1 2}}(i)\right|_{i=1, \ldots, L-1}$ and $\widetilde{\mathbf{w}}_{\mathbf{1 2}}=\left.\mathbf{w}_{\mathbf{1 2}}(i)\right|_{i=0, \ldots, L-2}$. These developments yield the final expression of the output signal distortion on $s_{1}(n)$

$$
\begin{aligned}
& \Delta s_{1}(n)=\Delta \mathbf{h}_{\mathbf{2 1}}{ }^{T}(n) \mathbf{s}_{\mathbf{2}}(n)- \\
& \mathbf{w}_{\mathbf{2 1}}{ }^{T}(n)\left[\widetilde{\mathbf{M}}_{\mathbf{1}}(n-1) \Delta \widetilde{\mathbf{h}}_{\mathbf{1 2}}(n)+\Delta \mathbf{S}_{\mathbf{1}}^{T}(n-1) \mathbf{w}_{\mathbf{1 2}}(n)\right] .
\end{aligned}
$$

Similar manipulations can be applied to the distortion in the second estimated signal $\widehat{s}_{2}(n)$ leading to

$$
\begin{aligned}
\Delta s_{2}(n)=\Delta \widetilde{\mathbf{h}}_{\mathbf{1 2}}^{T}(n) & \widetilde{\mathbf{s}}_{\mathbf{1}}(n-1)- \\
& \mathbf{w}_{\mathbf{1 2}}{ }^{T}(n) \mathbf{M}_{\mathbf{2}}^{T}(n-1) \Delta \mathbf{h}_{\mathbf{2 1}}(n-1)- \\
& \mathbf{w}_{\mathbf{1 2}}^{T}(n) \Delta \mathbf{S}_{\mathbf{2}}^{T}(n-1) \mathbf{w}_{\mathbf{2 1}}(n-1) \text {. (7) }
\end{aligned}
$$

Equations (6) and (7) define the output signal distortions associated with the feedback structure of Fig.1. The global network corresponding to these two relations is shown in Fig.2. Based on this network, the equilibrium point will be discussed in the next paragraph.

\section{B. Conservation of the distortion energy at each output}

Let us assume (A3) that the equilibrium state of the recursive structure given in Fig. 1 is obtained when the energy of the distortion on the output signal is the same on each channel, i.e. $E\left[\Delta s_{1}(n)^{2}\right]=E\left[\Delta s_{2}(n)^{2}\right]$. By considering that the filters are slowly time varying, i.e. $\mathbf{w}_{\mathbf{2 1}}(n) * \mathbf{w}_{\mathbf{1 2}}(n)=$ $\mathbf{w}_{\mathbf{1 2}}(n) * \mathbf{w}_{\mathbf{2 1}}(n-1)$, equal output distortion energy ensures identical correlation properties of the signals present at points
A and B (see Fig.2), which is equivalent to the equality of the power spectral densities given hereafter:

$$
\begin{aligned}
\Phi_{A A}\left(e^{j \omega}\right)=\sum_{k=-\infty}^{\infty} r_{A A}(k) e^{-j k \omega} & =\sum_{k=-\infty}^{\infty} r_{B B}(k) e^{-j k \omega} \\
& =\Phi_{B B}\left(e^{j \omega}\right)
\end{aligned}
$$

where $r_{A A}(k)=E[A(n) A(n+k)]$ is the correlation function of the signal in point A. Assuming (A4) that $s_{1}(n)$ and $s_{2}(n)$ are zero mean and independent, we get from Fig.2:

$$
\begin{gathered}
\Phi_{A A}\left(e^{j \omega}\right)=\Phi_{S_{1} S_{1}}\left(e^{j \omega}\right)\left|\widetilde{H}_{12}\left(e^{j \omega}\right)-\widetilde{W}_{12}\left(e^{j \omega}\right)\right|^{2}\left|W_{21}\left(e^{j \omega}\right)\right|^{2} \\
+\Phi_{S_{2} S_{2}}\left(e^{j \omega}\right)\left|H_{21}\left(e^{j \omega}\right)-W_{21}\left(e^{j \omega}\right)\right|^{2}
\end{gathered}
$$

The same reasoning holds in $\mathrm{B}$ with $r_{B B}(k)$. By introducing the misalignment vector, the power spectral densities can be rewritten as

$$
\begin{aligned}
\Phi_{A A}\left(e^{j \omega}\right)= & \Phi_{S_{1} S_{1}}\left(e^{j \omega}\right)\left|\Delta \widetilde{H}_{12}\left(e^{j \omega}\right)\right|^{2}\left|W_{21}\left(e^{j \omega}\right)\right|^{2}+ \\
& \Phi_{S_{2} S_{2}}\left(e^{j \omega}\right)\left|\Delta H_{21}\left(e^{j \omega}\right)\right|^{2} \\
\Phi_{B B}\left(e^{j \omega}\right)= & \Phi_{S_{2} S_{2}}\left(e^{j \omega}\right)\left|\Delta H_{21}\left(e^{j \omega}\right)\right|^{2}\left|W_{12}\left(e^{j \omega}\right)\right|^{2}+ \\
& \Phi_{S_{1} S_{1}}\left(e^{j \omega}\right)\left|\Delta \widetilde{H}_{12}\left(e^{j \omega}\right)\right|^{2} .
\end{aligned}
$$

Inserting (10) and (11) into (8), we get the following equilibrium relationship for the separation structure:

$$
\frac{\left|\Delta \widetilde{H}_{12}\left(e^{j \omega}\right)\right|^{2}}{\left|\Delta H_{21}\left(e^{j \omega}\right)\right|^{2}}=\frac{\Phi_{S_{2} S_{2}}\left(e^{j \omega}\right)}{\Phi_{S_{1} S_{1}}\left(e^{j \omega}\right)} \cdot \frac{\left|W_{12}\left(e^{j \omega}\right)\right|^{2}-1}{\left|W_{21}\left(e^{j \omega}\right)\right|^{2}-1} .
$$

Note that this relation is valid whatever the coefficients of the separating filters. Only stationarity assumptions are necessary.

\section{Signal to distortion ratio (SDR) analysis}

Through the above analysis, the signal to distortion ratio on each channel could be easily defined as follows:

$$
\begin{aligned}
\operatorname{SDR}_{1}\left(e^{j \omega}\right) & =\frac{\Phi_{S_{1} S_{1}}\left(e^{j \omega}\right)}{\Phi_{\Delta S_{1} \Delta S_{1}}\left(e^{j \omega}\right)} \\
& =\Phi_{S_{1} S_{1}}\left(e^{j \omega}\right) \frac{\left|1-W_{12}\left(e^{j \omega}\right) W_{21}\left(e^{j \omega}\right) e^{-j \omega}\right|^{2}}{\Phi_{A A}\left(e^{j \omega}\right)} \\
S_{D R_{2}}\left(e^{j \omega}\right) & =\frac{\Phi_{S_{2} S_{2}}\left(e^{j \omega}\right)}{\Phi_{\Delta S_{2} \Delta S_{2}}\left(e^{j \omega}\right)} \\
& =\Phi_{S_{2} S_{2}}\left(e^{j \omega}\right) \frac{\left|1-W_{12}\left(e^{j \omega}\right) W_{21}\left(e^{j \omega}\right) e^{-j \omega}\right|^{2}}{\Phi_{B B}\left(e^{j \omega}\right)} .
\end{aligned}
$$

Then, according to the stated equality principle expressed in (8), the following important relation holds:

$$
\frac{S D R_{1}\left(e^{j \omega}\right)}{S D R_{2}\left(e^{j \omega}\right)}=\frac{\Phi_{S_{1} S_{1}}\left(e^{j \omega}\right)}{\Phi_{S_{2} S_{2}}\left(e^{j \omega}\right)} .
$$

This relation describes the behavior of the recursive structure and is valid whatever the nature of the source signals and whatever the value of impulse responses of identification filters 


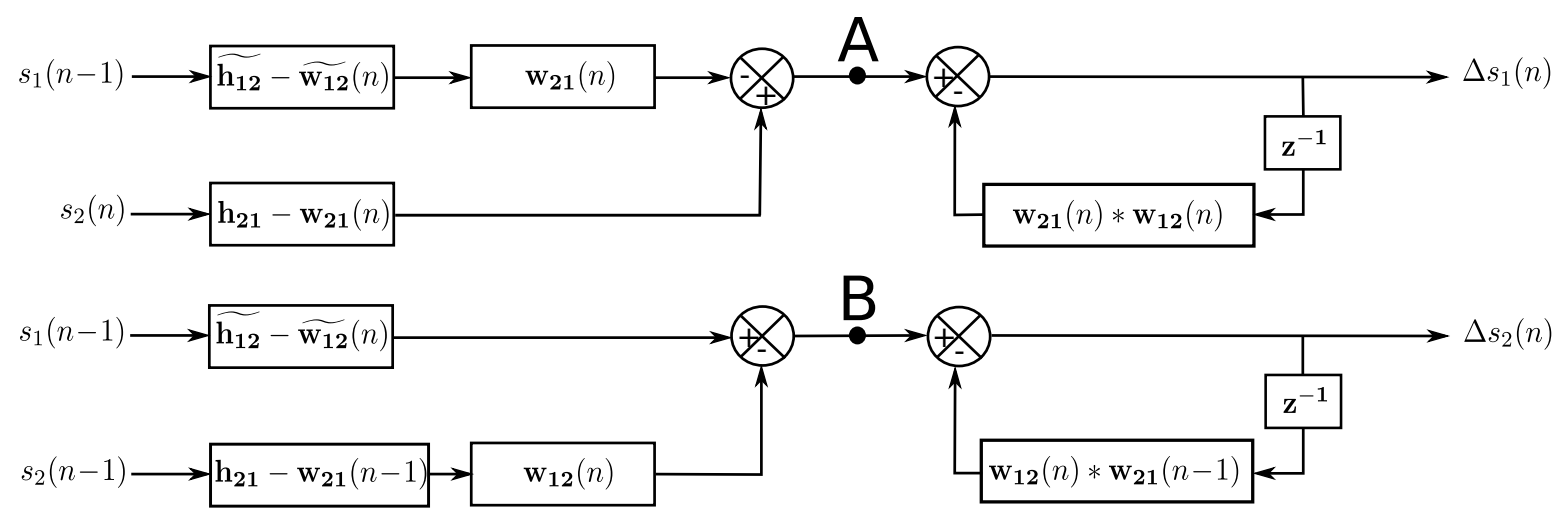

Fig. 2. Global network for getting the distortion signals on each channel.

$\mathbf{w}_{12}(n)$ and $\mathbf{w}_{21}(n)$. Note that if the two sources $s_{1}(n)$ and $s_{2}(n)$ correspond to white noises, with respective powers $\sigma_{s_{1}}^{2}$ and $\sigma_{s_{2}}^{2}$, the ratio given by (15) is equal to the average power ratio $\sigma_{s_{1}}^{2} / \sigma_{s_{2}}^{2}$ between the source signals.

\section{EXPERIMENTAL RESULTS}

\section{A. Experimental setup}

In this section we present simulation results illustrating the validity of the asymptotic analysis presented previously. To update the adaptive filters $\mathbf{w}_{\mathbf{1 2}}(n)$ and $\mathbf{w}_{\mathbf{2 1}}(n)$, we choose the symmetric adaptive decorrelation algorithm, [1]:

$$
\mathbf{w}_{\mathbf{j i}}(n+1)=\mathbf{w}_{\mathbf{j i}}(n)+\mu \widehat{s}_{i}(n) \widehat{\mathbf{s}}_{\mathbf{j}}(n) \quad \forall i \neq j \in 1,2
$$

where $\widehat{\mathbf{s}}_{\mathbf{j}}(n)=\left[\widehat{s}_{j}(n) \ldots \widehat{s}_{j}(n-L+1)\right]^{T}$ denotes the vector of the last $L$ output samples of the estimated signal $\widehat{s}_{j}$. We assume perfect modelisation with separating filters that have the same length as the mixing ones. In the experiments, coupling filters of length $L=20$ with (A5) the same energy are used and the separating filters are adapted from the relationship (16) with a low step size, $\mu=10^{-4}$. By fixing the same length for the two mixing filters we impose that the two sources are located in two halfplanes and thus only causal FIR filters are needed to achieve interference cancellation, as only delayed direct paths and reflections have to be modeled. The causality restrictions have consequences towards the physical interpretation of the signal model. Here, by shifting the impulse response associated with the source $s_{1}$ and sensor 2 such that $h_{12}^{0}=0$, the source $s_{1}$ is closer to sensor 1 than to sensor 2 and farther than one sample than source $s_{2}$ to sensor 1 . In addition, a necessary condition for correct implementation is that one of the zeroth-order coefficients of the adaptive and the generating filter equals zero

$$
w_{12}^{0}=h_{12}^{0}=0 \quad \text { or } \quad w_{21}^{0}=h_{21}^{0}=0 .
$$

Thus, by this assumption, we ensure inherent and structural stability [1] since no closed loops may exist without delay, i.e. $w_{12}^{0}(n) w_{21}^{0}(n)=0$. The input signal-to-noise ratios are computed using the ITU-T recommendation P.56 speech voltmeter (SV56). The speech signals are recorded from a male and a female speakers and shown in Fig. 3.

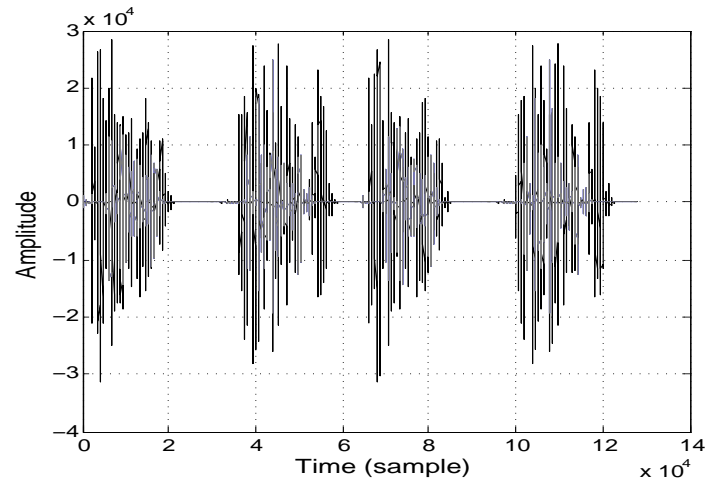

Fig. 3. Time-amplitude plot showing signal mixture.

\section{B. Equality principle and equilibrium relationship}

First we test the validity of our assumption (A3) in different SNR conditions and consider the previously established relations expressing the signal to distortion ratio at the output of each channel of the separation structure. In this paragraph, we assume that the source signals are the ones described previously. It is possible to compute, at each time instant $n$, the power spectral densities (8) and (9) by observing the value of the separating filters at that time instant. Once the power spectral densities $\Phi_{A A}\left(e^{j \omega}\right)$ and $\Phi_{B B}\left(e^{j \omega}\right)$ are known, we can then express, from relations (11) and (12), the value of the SDR on each spectral component of both channels. Such curves are displayed in Fig. 4, where the experimental and theoretical PSDs of the distortion of the output signals are plotted. The experimental PSDs are computed by observing the steady-state statistical properties of the distortion signals. To compute the theoretical PSDs expressed by

$$
\begin{aligned}
\Phi_{\Delta S_{1} \Delta S_{1}}\left(e^{j \omega}\right) & =\frac{\Phi_{A A}\left(e^{j \omega}\right)}{\left|1-W_{12}\left(e^{j \omega}\right) W_{21}\left(e^{j \omega}\right) e^{-j \omega}\right|^{2}} \\
\Phi_{\Delta S_{2} \Delta S_{2}}\left(e^{j \omega}\right) & =\frac{\Phi_{B B}\left(e^{j \omega}\right)}{\left|1-W_{12}\left(e^{j \omega}\right) W_{21}\left(e^{j \omega}\right) e^{-j \omega}\right|^{2}}
\end{aligned}
$$


where $\Phi_{A A}\left(e^{j \omega}\right)$ and $\Phi_{B B}\left(e^{j \omega}\right)$ are given in (8) and (11), we use the Fourier transform of the mean values $\overline{\mathbf{w}}_{\mathbf{1 2}}=$ $\lim _{n \rightarrow \infty} E\left[\mathbf{w}_{\mathbf{1 2}}(n)\right]$ and $\overline{\mathbf{w}}_{\mathbf{1 2}}=\lim _{n \rightarrow \infty} E\left[\mathbf{w}_{\mathbf{2 1}}(n)\right]$, which is equivalent to considering asymptotic behavior of the adaptive filters. The slight observed difference between the theoretical PSDs on each channel is due to the difference in the frequency content of the two speech sources and consequently reduces to zero in case of two synthetic white signals. We note the capability of the theoretical curves to closely predict the behavior of the experimental ones. The accuracy of the theoretical predictions tends to validate the assumption (A3) made in the theoretical analysis. Namely, at each time instant $n$, the steady state of the recursive structure is obtained when the condition of equal average power of distortion signals $E\left[\Delta s_{1}(n)^{2}\right]=E\left[\Delta s_{2}(n)^{2}\right]$ on each channel is checked.
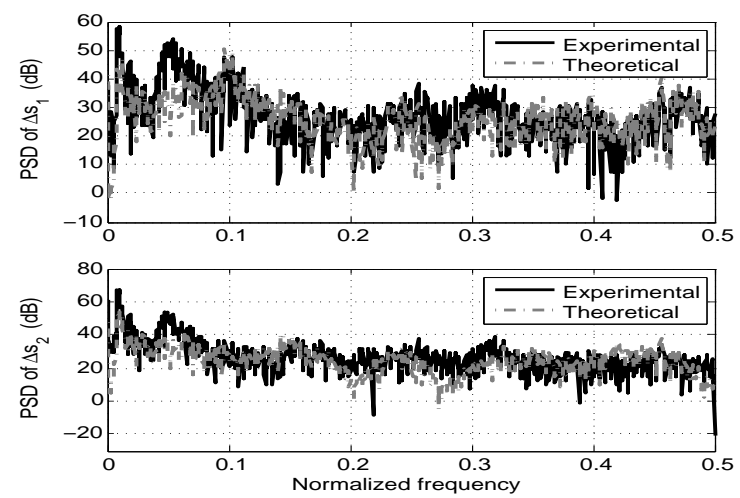

(a)
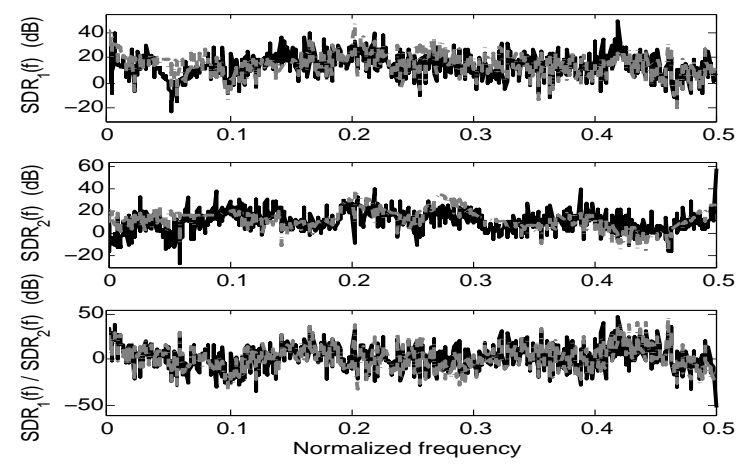

(b)

Fig. 4. Frequency behavior of the PSD of the distortion signals (a), and $S D R_{1}\left(e^{j \omega}\right), S D R_{2}\left(e^{j \omega}\right)$ and $S D R_{1}\left(e^{j \omega}\right) / S D R_{2}\left(e^{j \omega}\right)(\mathrm{b})$, with speech sources. Dashed curves denote the theoretical values and solid line the experimental ones. Results are obtained for an input SNR of $10 \mathrm{~dB}$.

Fig. 5 shows the ratio between the frequency average $S D R^{f b}$ of each channel. This one is obtained by integrating (15) over the whole spectral domain. Based on these results, we can see that as expected the evaluated ratio corresponds to the average power ratio of the speech sources across all SNRs considered. Consequently, the previous theoretical analysis and therefore the underlying assumptions are validated through experimental study.
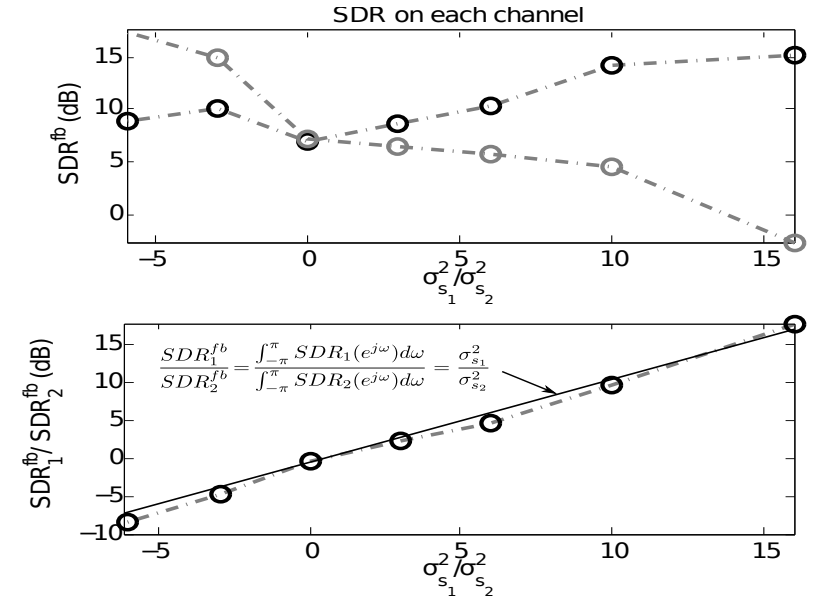

Fig. 5. Frequency average $S D R^{f b}$ obtained on channel 1 (black) and 2 (grey) with speech sources for different values of $\sigma_{s_{1}}^{2} / \sigma_{s_{2}}^{2}$.

\section{CONCLUSiON}

In this paper, the intrinsic convergence properties of the two-channel recursive structure have been discussed. Based on analysis of the relationship between output signals and their artifacts, we have shown that the equilibrium state is obtained when the energy of the distortion of the output signals is the same on each channel. Thus, in contrast with the usual approaches, this paper provides new relations for further analyzing the convergence and stability properties of the recursive adaptive structure of Fig. 1 independently of the used algorithm. Therefore, our approach is valid whatever the nature of the source signals (no assumptions on probability density functions of the sources) and whatever the value of the impulse responses as they check assumptions (A2) and (A5). Finally, experimental results show that the proposed equality principle applies to real mixture of speech signals.

\section{REFERENCES}

[1] S. V. Gerven and D. V. Compernolle, "Signal separation by symmetric adaptive decorrelation : stability, convergence and uniqueness," IEEE Transactions on Signal Processing, vol. 43, no. 7, pp. 1602-1612, July 1995.

[2] N. Charkani and Y. Deville, "Self-adaptive separation of convolutively mixed signals with a recursive structure. part i: stability analysis and optimization of asymptotic behaviour," Signal Processing, vol. 73, no. 3, pp. 225-254, March 1999.

[3] - "Self-adaptive separation of convolutively mixed signals with a recursive structure. part ii: theoretical extensions and application to synthetic and real signals," Signal Processing, vol. 75, no. 2, pp. 117-140, 1999.

[4] R. L. Zinser and J. B. Evans, "Some experimental and theoretical results using a new adaptive filter structure for noise cancellation in the presence of crosstalk," in IEEE International Conference on Acoustics, Speech and Signal Processing, vol. 10, April 1985, pp. 1253-1256.

[5] G. Mirchandani, R. L. Zinser, Jr., and J. B. Evans, "A new adaptive noise cancellation scheme in the presence of crosstalk," IEEE Transactions on circuits and systems II: Analog and Digital Signal Processing, vol. 39, no. 10, pp. 681-694, October 1992.

[6] L. Lepauloux, P. Scalart, and C. Marro, "An efficient low-complexity algorithm for crosstalk-resistant adaptive noise canceller," in European Signal Processing Conference, August 2009, pp. 204-208.

[7] M. Al-Kindi and J. Dunlop, "Improved adaptive noise cancellation in the presence of signal leakage on the noise reference channel," Signal Processing, vol. 17, no. 3, pp. 241-250, July 1989. 